\title{
A multi texture feature subset-based approach for Image classification
}

\author{
S Janardhanarao ${ }^{1}$, R Shankar ${ }^{2}$, Syed Inthiyaz ${ }^{3}$, Syed Shameem $^{4}$ \\ Professor $^{1}$, Associate Professor ${ }^{2,3,4}$ \\ ${ }^{1,2}$ Department of CSE, ${ }^{3,4}$ Department of ECE, Koneru Lakshmaiah Education Foundation, Vaddeswaram, Guntur.
}

\begin{abstract}
In artificial intelligence, pattern recognition of images is important aspect of computer visioning. The main objective is to identify the texture patterns based on the availability of features or the information. In this article multiple feature subset based on image classification which further depends on GLCM, Contrast visibility, Spatial Frequency, Energy of Gradients and histogram bins of binary patterns. The proposed method also presents a fusion based decision criterion for the texture classification. The experiments were conducted on real time datasets and the performance is evaluated with single and multiple feature subsets with SVM classifier. The identification parts will be tough due to small joints and occlusions. Identification of Image features is described in this paper with the help of Box Model Based Estimation, Speed up robust features and finally with Optical flow tracking algorithm.
\end{abstract}

Key words: Texture classification, Energy of gradients, SVM.

\section{INTRODUCTION}

In today's world images and graphics plays vital role in technology and they give accurate information to the people to better understand the world. Technology is increasing day by day in digital image processing with the help of internet and they are providing more images to people. On the other hand people are searching for effective and efficient image quality, indexing of images and methods of classification in digital world. Now a days Image classification became most popular theme in pattern recognition as well as in image mining.

In image classification generally in the computer the processing of particular images will be identified by the objects that were presented in that particular image. Most important application of texture is to identify the image regions by utilizing the texture characteristics. Person can easily recognize the vivid textures and its identities with different features of texture and primitives. In homogeneous images identifying the regions will be done by texture. Texture classification is done in computers by searching, browsing, identifying, comparison and classification of images from huge volume of digitalized images.
Texture classification aim is to generate the classification map of given image where each homogeneous texture region will be recognized by texture class and where it owns. In image processing and pattern identification, the most used approach is texture classification and it inspires the information about arrangement of spatial properties and fundamental elements of image [1].

This paper is systematized as below, section 1 presents the introduction to the current work, explaining the need and necessity of the work. Section 2 presents the existed works done by earlier researchers, its merits and demerits focusing on new outcomes. Section 3 presents background contents that were used in this work, section 4 presents the proposed approach with in detail explanation of the process of the proposed approach. Section 5 presents the experimental results obtained with current proposal ending with conclusions and discussions on proposed approach.

\section{RELATED WORK}

A large amount of research is conducted on distinguishing the texture for image classification a brief literature regarding the similar works proposed by earlier researchers are discussed in this section

In [2] Liu.et.al suggested the texture classification depends on the local frequency or local spatial representation. Here in the classification of texture authors used the spectral histogram as static feature. Spectral histograms contain the marginal distributions of bank of filters responses and encodes them indirectly local structure of pictures by filtering and gives good appearance through histogram state.

In [3] Mokhtari et.al states a novel rotation method in texture classification. Here in the given image color features were calculated by local binary pattern (LBP) and directional static features were evaluated by HOG (Histogram dominant of gradient) approach. Here image will be divided into bocks and each block gradient will be computed.

In [4] Arivazhagan and others suggested a new method to extract the features in texture classification. Here procedure of feature extraction is a hybrid model which also named as Dominant Neighborhood structure (DNS). To enhance the efficiency of dominant feature extraction this DNS model is combined with DWT feature set.

In [5] Boukhris et.al invented a new method in feature extraction which is called as (CDSDP) means Circular difference and statistical directional patterns here it combines the standard deviation and mean of the given image. 
In [6] Anish et.al, proposed multi stage decision based fusion approach for texture classification using global and local features with SVM classifier.

Much of the research algorithm were concentrated on extracting the single algorithm based feature extraction which may lack of some limitation however this can be overcome by incorporating multiple feature extraction algorithms. This paper focus on integrating multiple texture feature algorithms and a fusion based decisive approach is used for classification. The calculations which are included are LBP, Haar-course, Haar-course Open CV, Convolutional neural system[15] analyze is to provide and compare three perfectly computerized systems for rapid classification[16]

\section{METHODOLOGY}

\subsection{GLCM FEATURE SUBSET}

The Gray level co-occurrence matrix (GLCM) is a spatial dependent matrix which is calculated based on diverse orientations and separations among the pixel pairs in given image [7]. This can be mathematically represented as a function of $f(i, j, d, \Theta)$ where the pixel pair is separated by a distance of ' $d$ ' and is oriented at ' $\theta$ '. This is a square matrix which has value of the major pixel in given image. Describe how often gray levels are distributed and their spatial relationship in the image [8]. The features used in this work are (i) Skewness (ii) Kurtosis (iii) Contrast (iv) Homogeneity (v) Energy (vi) Correlation-coefficient and (vii) Inverse difference moment [9].

\subsection{SPATIAL FEATURES}

Contrast Visibility: It computes the deviancy of pixel from its mean value. The contrast of the image is defined mathematically as

$C V=\frac{1}{m x n} \sum_{i, j}^{N} \frac{|I(i, j)-\mu|}{\mu}$

Where $m$ and $n$ are the size of the image and $\mu$ denotes mean of the contrast values of given image.

Spatial frequency: It evaluates the image's activity level and computes the changes in frequency of rows and columns in image.

$$
\begin{gathered}
R F=\sqrt{\frac{1}{m x n} \sum_{i=1}^{m} \sum_{j=2}^{n}[I(i, j)-I(i, j-1)]^{2}} \\
C F=\sqrt{\frac{1}{m x n} \sum_{j=2}^{n} \sum_{i=1}^{n}[I(i, j)-I(i-1, j)]^{2}} \\
S F=\sqrt{R F^{2}+C F^{2}}
\end{gathered}
$$

Energy of Gradient: It is used to estimate the amount of focus in specific image. Let $\mathrm{fi}$ and $\mathrm{fj}$ be the energies calculated along rows and columns of the matrix then [10]

$$
E O G=\sum_{i=1}^{m-1} \sum_{j=1}^{n-1}\left(f_{i}^{2}+f_{j}^{2}\right)
$$

\subsection{HISTOGRAM OF LBP}

Local Binary Pattern (LBP) is the operator which extracts the scale and rotation invariant texture feature in an image. This operator is also termed as texture invariant operator with local neighborhood of image which was proposed by Ojala et.al in [11]. This operator is also a measurement for the local image contrast gray values which is supported with orthogonal measure. The LBP for a given matrix in local neighborhood can be obtained by substracting the centre element from the neighboring elements which are then multiplied by weights. 2D distributions of the LBP and local contrast measure were used as features.

\begin{tabular}{|l|l|l|}
\hline$i_{0}$ & $i_{1}$ & $i_{2}$ \\
\hline$i_{3}$ & $i_{4}$ & $i_{5}$ \\
\hline$i_{6}$ & $i_{7}$ & $i_{8}$ \\
\hline
\end{tabular}

(a)

Figure 1: (a) LBP operator binary sequence (b) weighted thresholds

So, this can be represented mathematically as

$$
\operatorname{LBP}\left(x_{c}, y_{c}\right)=\sum_{n=0}^{7} s\left(i_{n}-i_{c}\right) \cdot 2^{n}
$$

\subsection{PROPOSED METHOD}

The method proposed in this work is a multi-stage feature subset approach for the efficient image classification. This comprises of three feature subsets, in the first it calculates the GLCM features as stated in section 3. The second feature subset includes the spatial properties like contrast visibility, spatial frequency and Energy of gradients. Lastly the third feature subset includes the histogram of the texture extracted image obtained as the outcome of local binary pattern. These three merges together forma complex feature subset, which are classified using linear SVM classifier.

A decision-based approach is applied to enhance the classification rate. The three-feature subset and complex feature subset are grouped into three categories; the first two constitute the first category and later the rest of the subsets. The best of three categories are considered as the final classified output. The classification is evaluated with individual feature subset and also with the fusion based rule approach. The approach is tested with trained and non-trained image datasets and found that the proposed approach outperforms more even with untrained image dataset.

The experiments were conducted on Oliva Torralba Image Database available at [12]. The database consists of scenes of semantic categories with 256x256 resolution which are jpeg compressed color images [13]. In this work 200 images were considered for the evaluation of performance of the proposed 
approach. These images are partitioned into two and trained for multi class SVM [14],[15],[16],[17],[18],[19],[20],[21], [22],[23],[24]. Classification is performed on both trained and non-trained images and the performances are depicted below. It is observed from the experiments that the proposed fused based approach is yielding almost equal recognition rate of $89.9 \%$ for trained image dataset. But the algorithm outperforms with $56.4 \%$ with non-trained image dataset when the LBP approach could attain $54.2 \%$. These calculations reveal that the proposed approach could achieve better results when the feature are fused and follow the decision based rule for classifying.[25-29]

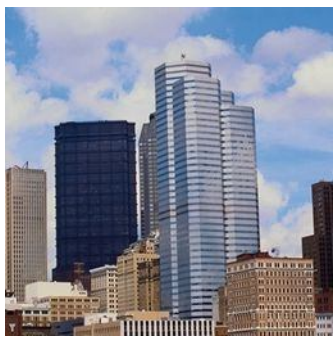

(a)

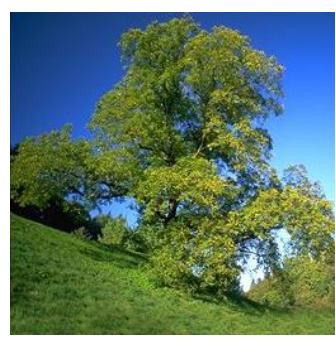

(c)

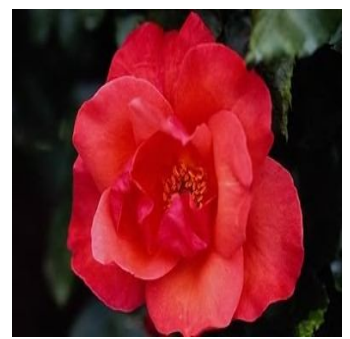

(e)

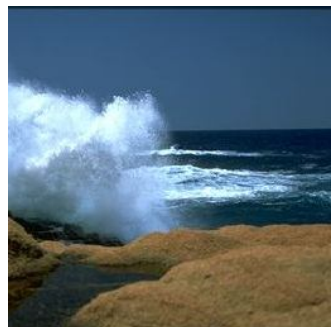

(b)

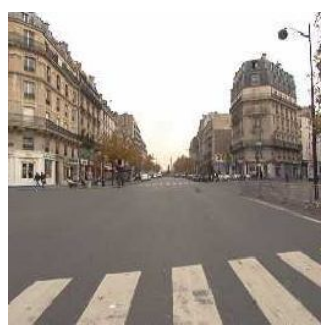

(d)

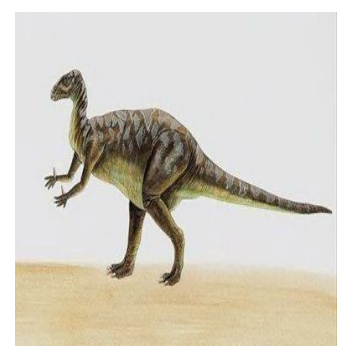

(f)
Figure 2: Different categories of images used for classification (a)buildings (b)coast (c) forest (d) street (e) flower (f) animals

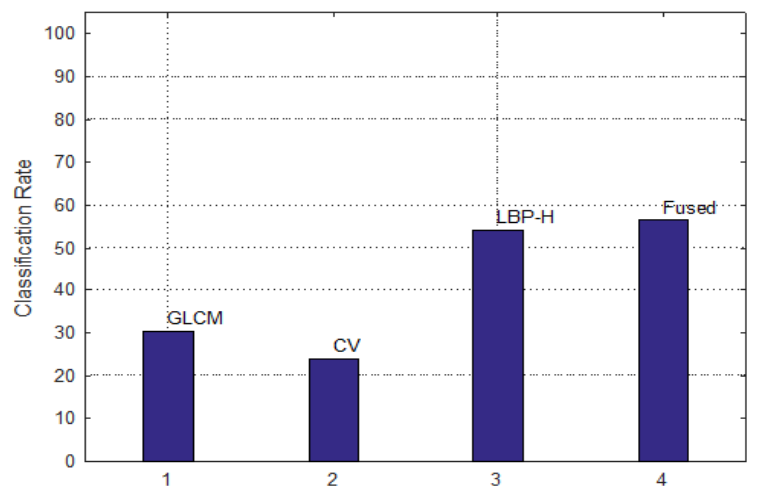

Figure 3: Performance analysis for non-trained image dataset

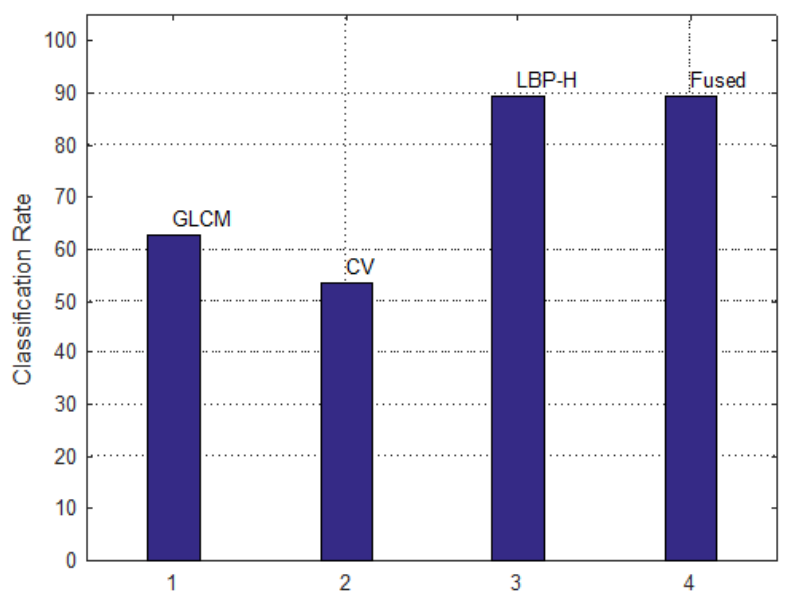

Figure 4: Performance analysis for trained image dataset

\section{CONCLUSION}

An efficient fused feature subset-based decision rule for image classification is proposed in this paper. The approach is compared against the individual feature subset performance and the proposed fusion based and from the experimental results it is revealed that the proposed decision rule-based approach is achieving better results with the traditional methods of classifying. In future, this work may be further moved with the inclusion of different classifiers and different binary patterns that yield better results.

\section{REFERENCES}

1. Minh N. Do and Martin Vetterli , Rotation Invariant Texture Characterization and Retrieval using Steerable Wavelet-domain Hidden Markov Models, IEEE transactions on multimedia ,Vol4-Issue 4,pg.no 517-527,2002

2. X. Liu and D. Wang, "Image and Texture Segmentation Using Local Spectral Histograms," in IEEE Transactions on Image Processing, vol. 15, no. 10, pp. 3066-3077, Oct. 2006.

3. M. Mokhtari, P. Razzaghi and S. Samavi, "Texture classification using dominant gradient descriptor," 2013 8th Iranian Conference on Machine Vision and Image Processing (MVIP), Zanjan, 2013, pp. 100-104

4. S. Arivazhagan and R. Benitta, "Texture classification using color local texture features," 2013 International Conference on Signal Processing, Image Processing \& Pattern Recognition, Coimbatore, 2013, pp. 220-223.

5. R. Boukhris Trabelsi, A. Damak Masmoudi and D. Sellami Masmoudi, "A new texture classification using circular difference and Statistical Directional Patterns," 2014 World Congress on Computer Applications and Information Systems (WCCAIS), Hammamet, 2014, pp. $1-5$.

6. A. C. Turlapaty, H. K. Goru and B. Gokaraju, "A Feature Subset Based Decision Fusion Approach for Scene Classification Using Color, Spectral, and Texture Statistics," 2017 IEEE 7th International Advance 
S Janardhanarao et al., International Journal of Emerging Trends in Engineering Research, 8(9), September 2020, 5824 - 5827

Computing Conference (IACC), Hyderabad, 2017, pp. 624-628.

7. David A Clausi, "An analysis of co-occurrence texture statistics as a function of grey level quantization," Canadian Journal of Remote Sensing, vol. 28, no. 1, pp. 45-62, 2002.

8. D. Haruka, A.Teruak, "Characterization of spatiotemporal stress distribution during food fracture by image texture analysis methods". Journal of Food Engineering, vol 81, pp.429-436, 2007.

9. A. R. Fallahi, M. Pooyan and H. Mohammadnejad, "Application of Morphological Operations in Human Brain CT Image With SVM," 2009 3rd International Conference on Bioinformatics and Biomedical Engineering, Beijing, 2009, pp. 1-4.

10. A. B. Siddiqui, M. A. Jaffar, A. Hussain and A. M. Mirza, "Block-Based Feature-Level Multi-Focus Image Fusion," 2010 5th International Conference on Future Information Technology, Busan, 2010, pp. 1-7.

11. Ojala $T$, Pietikainen $M \&$ Maenpa $T$, Multi-resolution gray-scale and rotation invariant texture classification with local binary patterns, IEEE Transactions on Pattern Analysis and Machine Intelligence, 24(7):971-987.2002 http://cvcl.mit.edu/database.htm

12. A. Oliva and A. Torralba. Modeling the shape of the scene: a holistic representation of the spatial envelope. IJCV, 42(3):145-175, 2001.

13. C.J.C._Burges,__ Laboratories, Lucent Technologies "A Tutorial on Support Vector Machines for Pattern Recogniton", Data Mining and Knowledge Discovery, vol 2, pp.121-167.1998

14. Anitha, R., Jyothi, S., Mandhala, V. N., Bhattacharyya, D., \& Kim, T. -. (2017). Deep learning image processing technique for early detection of alzheimer's disease. International Journal of Advanced Science and Technology, $\quad 107, \quad 85-104$. doi:10.14257/ijast.2017.107.07

15. Bangare, S. L., Pradeepini, G., \& Patil, S. T. (2017). Neuroendoscopy adapter module development for better brain tumor image visualization. International Journal of Electrical and Computer Engineering, 7(6), 3643-3654. doi:10.11591/ijece.v7i6.pp3643-3654

16. Jaya Lakshmi, R., \& Subba Rao, G. V. (2017). A re-.constructive algorithm to improve image recovery in compressed sensing. Journal of Theoretical and Applied

17. Nageswara Rao, M., \& Venu Gopala Rao, M. V. G. (2017). An hybrid feature selection technique classify glaucoma from fundus images. Journal of Advanced Research

18. Chakraborty, K., Si, T., De, A., \& Sharma, S. K. (2018). Clustering techniques for segmentation of soft tissue sarcoma in MR images. Journal of Advanced Research in

19. Malakonda Reddy, B., \& Ur Rahman, M. Z. (2018). Adaptive target extraction of synthetic aperture radar images using modified particle swarm optimization. Journal of Advanced Research in Dynamical and Control Systems, 10(9 Special Issue), 1347-1356. Retrieved from www.scopus.com
20. Nagaraju, G., Pardhasaradhi, P., \& Ghali, V. S. (2018). A new watermarking scheme for medical images with patient's details. International Journal of Engineering and

21. Prasad, M. V. D., Lakshmamma, B. J., Chandana, A. H., Komali, K., Manoja, M. V. N., Kumar, P. R., . . Kiran, P. S. (2018). An efficient classification of flower images

22. Reddy Gurunatha Swamy, P., \& Ananth Reddy, B. (2018). Human pose estimation in images and videos. International Journal of Engineering and Technology(UAE),//

23. Inthiyaz, S., Prasad, M.V.D., Usha Sri Lakshmi, R., Sri Sai, N.T.B., Kumar, P.P., Ahammad, S.H., "Agriculture based plant leaf health assessment tool: A deep learning perspective", International Journal of Emerging Trends in Engineering Research 7(11), pp. 690-694.

24. Myla, S., Marella, S.T., Goud, A.S., Ahammad, S.H., Kumar, G.N.S., Inthiyaz, S.," Design decision taking system for student career selection for accurate academic system',International Journal of Scientific and Technology Research8(9), pp. 2199-2206

25. Prasad, M.V.D., Inthiyaz, S., Teja Kiran Kumar, M., Sharma, K.H.S., Manohar, M.G., Kumari, R., Ahammad, S.H." Human activity recognition using deep learning", International Journal of Emerging Trends in Engineering Research 7(11), pp. 536-541.

26. Inthiyaz, S., Madhav, B.T.P., Kishore, P.V.V.” Flower image segmentation with PCA fused colored covariance and gabor texture features based level sets", Ain Shams Engineering Journal 9(4), pp. 3277-3291.

27. Inthiyaz, S., Kishore, P.V.V., Madhav, B.T.P.," Pre-informed level set for flower image segmentation" Smart Innovation, Systems and Technologies78, pp. 11-20.

28. Kishore, P. V. V.; Kumar, K. V. V.; Kumar, E. Kiran; Sastry, A. S. C. S.; Kiran, M. Teja; Kumar, D. Anil; Prasad, M. V. D.'Indian Classical Dance Action Identification and Classification with Convolutional Neural Networks",Advances in Multimedia, 2018,10.1155/2018/5141402.

29. Ahammad, SH; Rajesh, V; Rahman, MZU, "Fast and Accurate Feature Extraction-Based Segmentation Framework for Spinal Cord Injury Severity Classification" IEEE ACCESS, 2019,10.1109/ACCESS.2019.2909583. 